\title{
A Possible Role for Rusa Deer (Cervus timorensis russa) and Wild Pigs in Spread of Trypanosoma evansi from Indonesia to Papua New Guinea
}

\author{
SA Reid ${ }^{+}$, A Husein*, GW Hutchinson, DB Copeman
}

Department of Microbiology and Immunology, James Cook University of North Queensland, Townsville,

Australia *Department of Parasitology, Research Institute for Veterinary Science, Bogor, Indonesia

Movement of transmigrants and livestock from western Indonesia to southeastern areas of Irian Jaya near the border with Papua New Guinea may pose a risk of introducing Trypanosoma evansi into Papua New Guinea via feral Rusa deer (Cervus timorensis russa) and wild pigs which inhabit these areas in large numbers. Pilot experimental studies were conducted to observe infection in pigs and Rusa deer with a strain of $\mathrm{T}$. evansi isolated in Indonesia. Parasitaemia and signs of clinical disease were monitored each second day for 120 days. Trypanosomes were observed in haematocrit tubes at the plasma-buffy coat interface of jugular blood of deer and pigs on $86 \%$ and $37 \%$ of sampling occasions respectively. Parasitaemia was at a high level in deer for $35 \%$ of the time but for only $11.5 \%$ of the time in pigs. Results indicate that both Rusa deer and pigs have a high tolerance for infection with $\mathrm{T}$. evansi. The deer suffered mild anaemia evidenced by a $25 \%$ reduction in packed cell volume (PCV) 14 days after infection which coincided with the initial peak in parasitaemia. However, PCV had returned to pre infection values by the end of the experiment. The pigs showed no change in PCV. There were no visual indications of disease in either species and appetite was not noticeably affected. It was concluded that both Rusa deer and pigs were capable reservoir hosts for T. evansi but that Rusa deer, with their more persistent higher levels of parasitaemia, have more potential to spread T. evansi into Papua New Guinea from West Irian than pigs.

Key words: Trypanosoma evansi - reservoir hosts - epidemiology - pig - deer

Trypanosoma evansi is endemic throughout southeast Asia and the Indonesian archipelago with the possible exception of Irian Jaya. However, the movement of transmigrants and livestock from western Indonesia to areas in Irian Jaya near the border with Papua New Guinea over the past two decades may have introduced $T$. evansi. Since feral Rusa deer and wild pigs are common in southeastern areas of Irian Jaya and adjacent areas of the Western Province of Papua New Guinea, there is the possibility that the unrestricted movement of deer and pigs across the border in this region may introduce T. evansi to Papua New Guinea.

Pigs have been identified as an occasional host of $T$. evansi in Indonesia with rare outbreaks in commercial piggeries (Kraneveld \& Mansjoer 1947). Srivastava and Ahluwalia (1972) found that in pigs, trypanosomes were observed in blood

\footnotetext{
This study was supported by a grant from the Australian Centre for International Agricultural Research.

${ }^{+}$Corresponding author. Fax:61-7-47791526. E-mail: Simon.Reid@jcu.edu.au

Received 12 November 1998

Accepted 19 January 1999
}

smears 24-30 days after experimental infection with $T$. evansi but not thereafter even though blood was infective for mice. No reports have been found of differences between breeds of pigs in their susceptibility to infection with $T$. evansi whereas pathogenesis of infection in deer differs between species. Kraneveld and Mansjoer (1952, cited by Hoare 1972) found that T. evansi is highly pathogenic in Muntiacus muntjak and induces a chronic disease in Axis axis and Cervus timorensis. Furthermore, De Jesus (1963) showed T. evansi is particularly pathogenic to deer in the Philippines with death occurring eight weeks after infection.

Information on the clinical effects and pattern of parasitaemia of T. evansi in Rusa deer and pigs in Indonesia is incomplete. Knowledge of both factors is important when making an assessment of the potential of these hosts as reservoirs of infection with T. evansi. Accordingly, this information was sought in a pilot trial with pigs and Rusa deer.

\section{MATERIALS AND METHODS}

Four domestic pigs and two Rusa deer were infected with $5 \times 10^{8}$ trypanosomes from cryopreserved stabilates known to be highly pathogenic for rodents, held at the Research Institute for 
Veterinary Science, Bogor. Two additional pigs were not infected and acted as controls. Blood samples for parasitological examination by the haematocrit centrifugation technique (HCT) (Woo 1969) and estimation of packed cell volume (PCV) were taken every two days, haematological parameters were evaluated twice weekly for one month, and serum was collected every week for the 120 days duration of the experiment. The HCT results were expressed as an arbitrary score based on the numbers of trypanosomes observed in a single haematocrit tube. Animals were observed daily for signs of disease.

\section{RESULTS}

Parasites were detected by HCT in all infected animals within three days of infection. The pigs showed a rapid rise in parasitaemia, peaking at ten days then progressively declining. After one month occasional peaks of parasitaemia were observed at intervals of up to 20 days. Parasitaemia also rose rapidly over the first week in deer but, unlike the pattern in pigs, it remained high for 12 weeks before adopting a fluctuating pattern of repeated peaks and troughs. Deer had higher and more persistent peaks of parasitaemia than pigs; trypanosomes were observed (with HCT) in deer on $86 \%$ of sampling occasions but only on $37 \%$ in pigs, and parasitaemia was high in deer for $35 \%$ of the time but for only $11.5 \%$ of the time in pigs. The deer suffered mild anaemia evidenced by a $25 \%$ reduction in PCV 14 days after infection, which coincided with the initial peak in parasitaemia. However, PCV had returned to pre-infection values by the end of the experiment. The pigs showed no change in PCV. There were no visual indications of disease in either species and appetite was not noticeably affected.

Data on levels and duration of parasitaemia from this experiment have been combined with results from experimental infection in cattle and buffalo conducted at the Research Institute for Veterinary Science under similar conditions (Partoutomo 1993). Results are presented in the Table. It may be seen that the proportion of sampling occasions on which animals were negative or at a particular level of parasitaemia was similar in pigs, buffalo and Friesian cattle. In contrast, Rusa deer were parasite-negative for approximately one third the number of occasions and highly parasitaemic for approximately twice the number of occasions as the other species.

\section{DISCUSSION}

The persistent high levels of parasitaemia in Rusa deer and their tolerance to infection with T. evansi suggest that these animals may be good

\section{TABLE}

Comparison of parasitaemia in Rusa deer, pigs, buffalo and Friesian cattle observed each second day for 120 days after experimental infection with Trypanosoma evansi

Mean percentage of total number of occasions samples were examined

Trypanosomes/

\begin{tabular}{crrrr} 
HCT tube & Deer & Pig & Buffalo $^{a}$ & Friesian $^{a}$ \\
\hline 0 & 14.4 & \multicolumn{1}{c}{63} & 47.3 & 59.7 \\
$1-5$ & 29.8 & 17.8 & 18.1 & 18.8 \\
$6-10$ & 12.5 & 2.9 & 7.5 & 2.6 \\
$11-20$ & 8.7 & 4.8 & 3.3 & 2.1 \\
$>20$ & 34.6 & 11.5 & 23.8 & 16.8 \\
\hline
\end{tabular}

$a$ : data modified from information presented by Partoutomo (1993).

reservoirs of infection for other animals in their vicinity. During the wet season, large numbers of Rusa deer are crowded together with large numbers of wallabies (Macropus agilis) and wild pigs on small areas above flood height on the coastal plain, which is continuous in the southwest of the Western Province of PNG and the southeast of Irian Jaya. Since the wet season is also favourable for tabanids (I Owen, pers. comm.), such conditions could be expected to promote the spread of $T$. evansi between deer and wild pigs, and also to wallabies if they are susceptible. Movement of these animals is unrestricted by man-made or significant physical barriers in the border region during the dry season. This introduces the possibility that infection, spread to deer from domestic animals brought to the southeast of Irian Jaya from areas of Indonesia endemic for T. evansi, may be carried to Western Province, where it may be expected to persist in the Rusa deer and wild pigs. However, this may not pose a significant threat to domestic animals in PNG as few are present in Western Province and the Fly River is a substantial barrier, likely to confine the disease, if it is introduced to the sparsely populated region west of the river. This situation may already exist as a recent parasitological and serological survey of 252 cattle from an area to the east of the southern reaches of the Fly River revealed no evidence of infection with $T$. evansi whereas 4 of 18 sera collected from Rusa deer near the southwest border areas with Irian Jaya were strongly positive on the card agglutination test for trypanosomiasis (CATT, Institute of Tropical Medicine, Laboratory of Serology, Nationalestraat 155, B-2000 Antwerp, Belgium).

There are limits to which information derived from the small number of experimental animals in this trial may be interpreted but the uniformity of 
results within the infected pigs and deer give a degree of confidence that the results are repeatable. This being so, it appears that pigs, Friesian cattle and swamp buffaloes all have a similar pattern of parasitaemic and non-parasitaemic days during the first few months after infection, in contrast to Rusa deer where there are substantially fewer days when no parasites are present, and many more days when parasitaemia is high. The inference is that Rusa deer may be a more effective host for transmission of $T$. evansi than pigs, Friesian cattle or swamp buffaloes, but such a conclusion takes no account of other determinants such as attractiveness of deer for tabanids and their herding behaviour, the territory over which they range or the proximity of their association with domestic animals.

\section{ACKNOWLEDGEMENTS}

To Mr K Riroriro, Mr B Laba, Mr D Siswansyah and Mr M Dahlan for technical assistance. To the cooperation in use of facilities of Dr Sjamsul Bahri and Dr Sutijono Partoutomo at the Research Institute for Veterinary Science, Bogor and Dr Ilagi Puana at the National Veterinary Laboratory in Port Moresby.

\section{REFERENCES}

De Jesus Z 1963. Peculiarities and importance of Surra infection in reservoir hosts. Philippine J Vet. Med 2: 1-14.

Hoare CA 1972. The Trypanosomes of Mammals. A Zoological Monograph, Blackwell Scientific Publications, Oxford, UK, 748pp.

Kraneveld FC, Mansjoer M 1947. Surra in pigs. NedInd Blad Diergeneesk 54: 254-261.

Kraneveld FC, Mansjoer M 1952. Onderzoekingen oner de gevoeligheod voor Surra. II. Het verloop der ziekte bij enkele in het wild levende dieren in Indonesi. Hemera Zoa 59: 117.

Partoutomo S 1993. Studies on the Epidemiology of Trypanosoma evansi in Java, PHD thesis, Department of Biomedical and Tropical Veterinary Science, James Cook University, North Queensland, Townsville.

Srivastava RP, Ahluwalia SS 1972. Clinical observations on pigs experimentally infected with Trypanosoma evansi. Indian Vet J 49: 1186-1186.

Woo PTK 1969. The haematocrit centrifuge for the detection of trypanosomes in blood. Can J Zool 47: 921-923. 
Rusa Deer and Wild Pigs in Spread of T. evansi • SA Reid et al. 